\title{
Electroweak naturalness in the three-flavor type I seesaw model and implications for leptogenesis
}

\author{
Jackson D. Clarke, Robert Foot, and Raymond R. Volkas \\ ARC Centre of Excellence for Particle Physics at the Terascale, School of Physics, \\ University of Melbourne, Parkville, Victoria 3010, Australia \\ (Received 12 February 2015; published 10 April 2015)
}

\begin{abstract}
In the type I seesaw model, the naturalness requirement that corrections to the electroweak $\mu$ parameter not exceed $1 \mathrm{TeV}$ results in a rough bound on the lightest right-handed neutrino mass, $M_{N_{1}} \lesssim 3 \times 10^{7} \mathrm{GeV}$. In this paper we derive generic bounds applicable in any three-flavor type I seesaw model. We find $M_{N_{1}} \lesssim 4 \times 10^{7} \mathrm{GeV}$ and $M_{N_{2}} \lesssim 7 \times 10^{7} \mathrm{GeV}$. In the limit of one massless neutrino, there is no naturalness bound on $M_{N_{3}}$ in the Poincaré protected decoupling limit. Our results confirm that no type I seesaw model can explain the observed neutrino masses and baryogenesis via hierarchical $\left(N_{1^{-}}, N_{2^{-}}\right.$, or $N_{3}$-dominated) thermal leptogenesis while remaining completely natural.
\end{abstract}

DOI: $10.1103 /$ PhysRevD.91.073009

PACS numbers: 14.60.Pq, 11.30.Fs, 14.60.St, 98.80.Cq

\section{INTRODUCTION}

The discovery of a particle consistent with the standard model (SM) Higgs Boson [1] appears to confirm the standard mechanism of electroweak spontaneous symmetry breaking: The Higgs field $\phi$ gains a vacuum expectation value $\langle\phi\rangle$ by virtue of the potential $V=-\mu^{2}|\phi|^{2}+\lambda|\phi|^{4}$ at a scale set by the renormalized parameter $\mu=m_{h} / \sqrt{2} \approx$ $88 \mathrm{GeV}$. Much of modern high energy physics has been concerned with the naturalness of this scale. The fact remains that the SM alone (without gravity) suffers no hierarchy problem; a large cancellation between an unmeasurable bare parameter and an unphysical cutoff scale can be assigned no physical significance. Indeed, if no physical large scale exists there can be no hierarchy problem. Neither is the presence of such a scale sufficient for a hierarchy problem $[2,3] .{ }^{1}$ To find out, the pragmatic physicist should just take a model, explicitly calculate corrections, and express them in terms of (in principle) measurable parameters. If those corrections are large compared to measured values, only then could naturalness become a concern.

Vissani did just that in the one-flavor type I seesaw model [4] (see also Refs. [5]). He calculated a correction to the electroweak $\mu$ parameter

$$
\delta \mu^{2} \approx \frac{1}{4 \pi^{2}} \frac{1}{\langle\phi\rangle^{2}} m_{\nu} M_{N}^{3}
$$

where $\langle\phi\rangle \approx 174 \mathrm{GeV}$. If required to be less than $1 \mathrm{TeV}^{2}$, a neutrino of mass $m_{\text {atm }} \approx 0.05 \mathrm{eV}$ implies an upper bound on the right-handed neutrino mass, $M_{N} \lesssim 3 \times 10^{7} \mathrm{GeV}$. Nevertheless, the model still provides an elegant explanation of the smallness of the neutrino mass scale [6]. The type I seesaw model is also capable of explaining

\footnotetext{
${ }^{1}$ See Ref. [3] for citations to other literature on this topic.
}

baryogenesis via leptogenesis, the Fukugita-Yanagida mechanism [7]. The standard version requires the lightest right-handed neutrino to satisfy $M_{N_{1}} \gtrsim 5 \times 10^{8} \mathrm{GeV}[8,9]$, in obvious tension with the naturalness bound above.

Thus it appears that one cannot use the type I seesaw model to explain both the observed neutrino masses and baryogenesis via standard thermal leptogenesis without ceding naturalness. This paper aims to establish whether this conclusion holds in the three-flavor type I seesaw model in full generality.

\section{PRELIMINARIES}

The pertinent part of the type I seesaw Lagrangian is

$-\mathcal{L}=\overline{l_{L}^{i}} y_{e}^{i j} e_{R}^{j} \phi+\overline{l_{L}^{i}} y_{\nu}^{i j} \nu_{R}^{j} \tilde{\phi}+\frac{1}{2} \overline{\left(\nu_{R}^{i}\right)^{c}} M_{N}^{i j} \nu_{R}^{j}+$ H.c.,

where $i, j$ are flavor indices, $l_{L}^{i} \equiv\left(\nu_{L}^{i}, e_{L}^{i}\right)^{T}$, and $\tilde{\phi} \equiv i \tau_{2} \phi^{*}$. One is free to rotate and rename the fields such that $M_{N} \equiv$ $\mathcal{D}_{M}=\operatorname{diag}\left(M_{1}, M_{2}, M_{3}\right)$ has real positive diagonal entries.

After symmetry breaking, and if $M_{j} \gg y_{\nu}^{i j}\langle\phi\rangle$, the Lagrangian becomes

$-\mathcal{L} \approx \overline{l_{L}^{i}} y_{e}^{i j} e_{R}^{j}\langle\phi\rangle+\frac{1}{2} \overline{\nu_{L}^{i}} m_{\nu}^{i j}\left(\nu_{L}^{j}\right)^{c}+\frac{1}{2} \overline{\left(\nu_{R}^{i}\right)^{c}} M_{i} \nu_{R}^{i}+$ H.c.,

where $m_{\nu}=\langle\phi\rangle^{2} y_{\nu} \mathcal{D}_{M}^{-1} y_{\nu}^{T}$ is the neutrino mass matrix. One can diagonalize $m_{\nu}$ with a unitary matrix $U$,

$$
\mathcal{D}_{m} \equiv \operatorname{diag}\left(m_{1}, m_{2}, m_{3}\right)=U m_{\nu} U^{T},
$$

where $m_{i}$ are the neutrino masses. Following Casas-Ibarra [10], it is possible to express $y_{\nu}$ as 


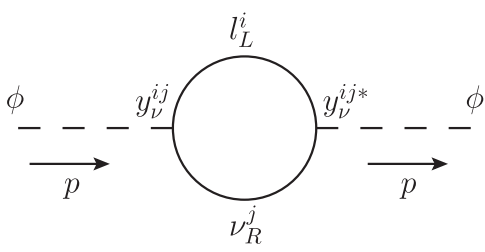

FIG. 1. Loop diagram leading to $\delta \mu^{2}$.

$$
y_{\nu}=\frac{1}{\langle\phi\rangle} U^{\dagger} \mathcal{D}_{m}^{\frac{1}{2}} R \mathcal{D}_{M}^{\frac{1}{2}},
$$

where $R$ is a (possibly complex) orthogonal $\left(R^{T} R=R R^{T}=\square\right)$ matrix. $R$ is physically relevant and measurable in principle (e.g. by studying the production and decays of the $\nu_{R}^{j}$ ), however measurements to date tell us nothing about it.

From Fig. 1, we calculate the correction to $\mu^{2}$ in $\overline{\mathrm{MS}}$ scheme as

$$
\left|\delta \mu^{2}\right|=\frac{1}{4 \pi^{2}} y_{\nu}^{i j} M_{j}^{2}\left|\log \left[\frac{M_{j}}{\mu_{R}}\right]-\frac{1}{4}\right| y_{\nu}^{i j *}+\mathcal{O}\left(\mu^{2}\right),
$$

where $\mu_{R}$ is the renormalization scale. The renormalization group equation for $\mu^{2}\left(\mu_{R}\right)$ will receive a contribution $\approx \frac{1}{4 \pi^{2}}\left|y_{\nu}^{i j}\right|^{2} M_{j}^{2}$. If this contribution is much larger than the electroweak scale, only a very finely tuned $\mu^{2}\left(\mu_{R} \gg M_{j}\right)$ will achieve $m_{h} \sim 125 \mathrm{GeV}$; the natural scale for $m_{h}$ is $\sim\left|y_{\nu}^{i j}\right| M_{j}$.

Taking the quantity within absolute values to be unity, the contribution from all nine diagrams becomes

$$
\left|\delta \mu^{2}\right| \approx \frac{1}{4 \pi^{2}} \operatorname{Tr}\left[y_{\nu} \mathcal{D}_{M}^{2} y_{\nu}^{\dagger}\right] .
$$

Upon substitution of the Casas-Ibarra form [Eq. (5)], one obtains the simple relation

$$
\left|\delta \mu^{2}\right| \approx \frac{1}{4 \pi^{2}} \frac{1}{\langle\phi\rangle^{2}} \operatorname{Tr}\left[\mathcal{D}_{m} R \mathcal{D}_{M}^{3} R^{\dagger}\right] .
$$

Note that there is no explicit dependence on $U$, as one could anticipate, since all of $U$ can be absorbed by $l_{L} \rightarrow U l_{L}$, $y_{\nu} \rightarrow U y_{\nu}$. One ends up with three positive-definite corrections proportional to the cube of each heavy neutrino mass. Naturalness demands that these corrections each be less than some scale not far above $\mu \approx 88 \mathrm{GeV}$. In our calculations we therefore require the three bounds:

$$
\begin{aligned}
& \frac{1}{4 \pi^{2}} \frac{1}{\langle\phi\rangle^{2}} M_{j}^{3} \sum_{i} m_{i}\left|R_{i j}\right|^{2}<1 \mathrm{TeV}^{2}, \\
\Rightarrow & M_{j} \lesssim 2.9 \times 10^{7} \mathrm{GeV}\left(\frac{0.05 \mathrm{eV}}{\sum_{i} m_{i}\left|R_{i j}\right|^{2}}\right)^{\frac{1}{3}},
\end{aligned}
$$

where $R_{i j}$ are the entries of $R$. Our results can be easily rescaled for a different naturalness criterion.

\section{RESULTS}

Equation (9) results in three upper bounds on the righthanded neutrino masses. It says nothing about their mass ordering, since one can always append to $R$ a permutation matrix. However we can always order the bounds by their size; we call them $B_{j}$ and take $B_{1} \leq B_{2} \leq B_{3}$.

We are interested in the values of $B_{j}$ attainable from Eq. (9). Thus all we have to do is extremize these bounds over $R$. We used the mass squared differences of NuFIT v2.0 [11],

$$
\begin{aligned}
& \Delta m_{21}^{2}=7.50 \times 10^{-5} \mathrm{eV}^{2}, \\
& \Delta m_{3 l}^{2}= \pm 2.46 \times 10^{-3} \mathrm{eV}^{2},
\end{aligned}
$$

where $\Delta m_{3 l}^{2}=\Delta m_{31}^{2}>0$ for normal ordering (NO) and $\Delta m_{3 l}^{2}=\Delta m_{32}^{2}<0$ for inverted ordering (IO), and treat the lightest neutrino mass $\left(m_{1}\right.$ for $\mathrm{NO}$ or $m_{3}$ for IO) as unknown. The $B_{j}$ were numerically extremized over a parametrization of $R$. The results were checked analytically and with scatterplots. Figures $2-3$ show the case for NO and IO when $R$ is real. The solid lines are for $R=\rrbracket$.

The first thing to notice is that as the lightest neutrino mass tends to zero, the largest bound $B_{3}$ can potentially evaporate. This only happens in models where $R$ is of a particular form, e.g. in NO, as is evident from Eq. (9),

$$
R=\left(\begin{array}{ccc}
R_{11} & R_{12} & \pm 1 \\
R_{21} & R_{22} & 0 \\
R_{31} & R_{32} & 0
\end{array}\right),
$$

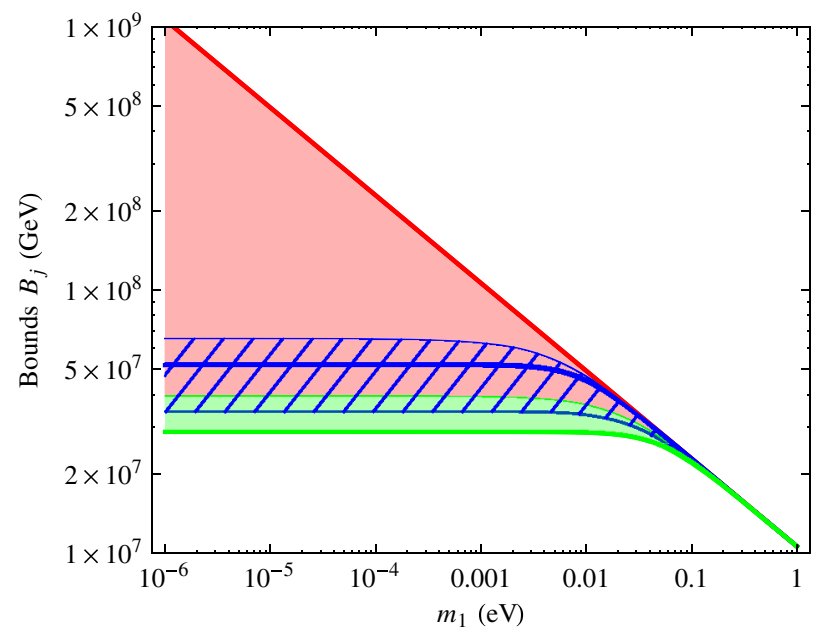

FIG. 2 (color online). As a function of the lightest neutrino mass in normal ordering (NO), shown as darker/hatched/lighter (red/blue-hatched/green color) is the region of attainable values for the $B_{3} \geq B_{2} \geq B_{1}$ upper bounds on right-handed neutrino masses, by requiring that the corrections to the electroweak $\mu$ parameter be no greater than $1 \mathrm{TeV}$ [Eq. (9)]. The regions assume the orthogonal matrix $R$ is real. Thick solid lines show the case when $R=\rrbracket$. The case for complex $R$ is similar, except there is no lower limit to the $B_{j}$ (see text). 


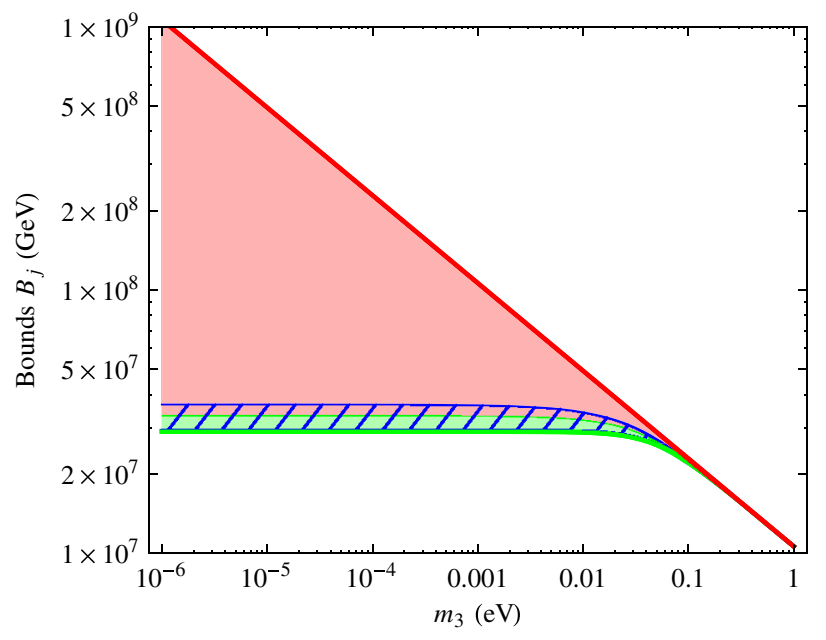

FIG. 3 (color online). As in Fig. 2 but as a function of the lightest neutrino mass in inverted ordering (IO). Note that the thick blue line is obscured by the thick green line.

or some column permutation, where $R_{11}=R_{12}=0$ if $R$ is real. This corresponds to the Poincaré protected decoupling limit $y_{\nu}^{i 3} \rightarrow 0$ and an effective two-flavor seesaw [12].

The maximization of $B_{2}$ occurs when $B_{2}=B_{3}$ and corresponds in NO to $R$ of the form

$$
R= \pm\left(\begin{array}{ccc}
0 & \frac{1}{\sqrt{2}} & -\frac{1}{\sqrt{2}} \\
0 & \frac{1}{\sqrt{2}} & \frac{1}{\sqrt{2}} \\
\pm 1 & 0 & 0
\end{array}\right)
$$

up to column permutations. Similarly the minimization of $B_{2}$ occurs when $B_{1}=B_{2}$, and corresponds to

$$
R= \pm\left(\begin{array}{ccc}
0 & 0 & \pm 1 \\
\frac{1}{\sqrt{2}} & -\frac{1}{\sqrt{2}} & 0 \\
\frac{1}{\sqrt{2}} & \frac{1}{\sqrt{2}} & 0
\end{array}\right)
$$

The maximization (minimization) of $B_{1}\left(B_{3}\right)$ occurs when $B_{1}=B_{2}=B_{3}$. This corresponds to a conspiratorial form of $R$.

Even though these arrangements are possible, it is clear from Figs. 2-3 that it is not possible to construct a type I seesaw model that changes the bounds $B_{1}$ and $B_{2}$ by more than a factor of 2 when $R$ is real. Even if one does saturate these bounds, it is not possible then to place the righthanded neutrino masses at this bound and maintain a hierarchy that is the basis of many of the calculations for thermal leptogenesis.

In the case of $R$ complex the upper limits of the $B_{j}$ are the same as the $R$ real case. However the lower limits can potentially be much lower. The reason is that complex $R$ with entries of arbitrarily large magnitude exist. Let us illustrate this in the two-flavor case. An example is

$$
R=\left(\begin{array}{cc}
\cosh x & i \sinh x \\
-i \sinh x & \cosh x
\end{array}\right)
$$

In this case,

$U y_{\nu}=\frac{1}{\langle\phi\rangle}\left(\begin{array}{cc}\sqrt{m_{1} M_{1}} \cosh x & -i \sqrt{m_{1} M_{2}} \sinh x \\ i \sqrt{m_{2} M_{1}} \sinh x & \sqrt{m_{2} M_{2}} \cosh x\end{array}\right)$.

If $\cosh x \gg 1$, one need only calculate $m_{\nu}$ to see that the smallness of neutrino masses is only explained by fortuitous cancellations between entries of $y_{\nu}$ that constitute a fine-tuning. If we demand that the entries of $R$ have magnitude not exceeding 1 , then the results for complex $R$ are essentially the same as in the real case. In general, however, allowing complex $R$ can only degrade the attainable region for the $B_{j}$.

\section{CONCLUSION}

Corrections to the $\mu$ parameter in the type I seesaw model can be expressed in the concise form of Eq. (8), as a function of an unknown (but in principle measurable) orthogonal matrix $R$. Requiring these corrections to be less than $1 \mathrm{TeV}$ results in three bounds on the right-handed neutrino masses, $B_{1} \leq B_{2} \leq B_{3}$. As shown in Figs. 2-3, we find that $B_{1}$ and $B_{2}$ can be varied by no more than a factor 2 around their values in the $R=\rrbracket$ case. The bound $B_{3}$ evaporates in models with a massless neutrino and $R$ of the form of Eq. (11) (up to column permutations) for NO, and similarly for IO. This corresponds to the Poincare protected decoupling limit $y_{\nu}^{i 3} \rightarrow 0$. In short, we obtain the generic bounds

$$
\begin{aligned}
& M_{N_{1}} \lesssim 4 \times 10^{7} \mathrm{GeV}, \\
& M_{N_{2}} \lesssim 7 \times 10^{7} \mathrm{GeV}, \\
& M_{N_{3}} \lesssim 3 \times 10^{7} \mathrm{GeV}\left(\frac{0.05 \mathrm{eV}}{m_{\min }}\right)^{\frac{1}{3}},
\end{aligned}
$$

where $m_{\min }$ is the lightest neutrino mass. For a given model, however, the bounds will be more stringent.

Baryogenesis via standard $\left(N_{1}\right.$-dominated, hierarchical) thermal leptogenesis requires $M_{N_{1}} \gtrsim 5 \times 10^{8}\left(2 \times 10^{9}\right) \mathrm{GeV}$ for $N_{1}$ with thermal (zero) initial abundancy [9], ${ }^{2}$ in conflict with Eq. (16a).

In $N_{2}$ leptogenesis, it is possible to have $M_{N_{1}} \lesssim 10^{7} \mathrm{GeV}$. There are two scenarios. One is in the $N_{1}$-decoupling limit [14], and the other relies on special flavor alignments to protect an $N_{2}$-generated asymmetry from $N_{1}$ washout [15]. Both are in conflict with Eq. (16b), as such a light $N_{2}$ is

\footnotetext{
${ }^{2}$ These bounds are unaffected by flavor considerations [13].
} 
unable to produce the required asymmetry for the usual reasons $[8,9]$.

One might think that there is still room left for $N_{3}$ leptogenesis. This turns out to not be the case. In order to naturally have $M_{N_{3}} \gtrsim 10^{9} \mathrm{GeV}$, one must have $m_{\min } \lesssim$ $10^{-6} \mathrm{eV}$ and $R$ in a decoupling limit such as Eq. (11). However in this limit the charge parity asymmetry from $N_{3}$ decays is [14]

$$
\varepsilon_{3} \sim 10^{-1} \sum_{i=1,2} \frac{m_{\min } M_{i}^{2}}{\langle\phi\rangle^{2} M_{3}} \operatorname{Im}\left(R_{1 i}^{2}\right),
$$

which is far too small.
Thus our results confirm that no minimal type I seesaw model can explain the neutrino masses and baryogenesis via hierarchical $\left(N_{1^{-}}, N_{2^{-}}\right.$, or $N_{3}$-dominated) thermal leptogenesis while remaining completely natural. In the minimal scenario, the only ways to avoid this conclusion are to assume $N_{1}$ has dominant initial abundancy [in this case leptogenesis is possible with $M_{N_{1}} \gtrsim 2 \times 10^{7} \mathrm{GeV}$ [9], which is marginally consistent with Eq. (16a)], allow a resonant enhancement [16], or consider an entirely different mechanism [17].

\section{ACKNOWLEDGMENTS}

This work was supported in part by the Australian Research Council.
[1] G. Aad et al. (ATLAS Collaboration), Phys. Lett. B 716, 1 (2012); S. Chatrchyan et al. (CMS Collaboration), Phys. Lett. B 716, 30 (2012).

[2] W. A. Bardeen, Report Nos. FERMILAB-CONF-95-391-T, C95-08-27.3, 1995.

[3] R. Foot, A. Kobakhidze, K. L. McDonald, and R. R. Volkas, Phys. Rev. D 89, 115018 (2014).

[4] F. Vissani, Phys. Rev. D 57, 7027 (1998).

[5] J. Casas, J. Espinosa, and I. Hidalgo, J. High Energy Phys. 11 (2004) 057; A. Abada, C. Biggio, F. Bonnet, M. Gavela, and T. Hambye, J. High Energy Phys. 12 (2007) 061; Z.-z. Xing, Prog. Theor. Phys. Suppl. 180, 112 (2009); F. Bazzocchi and M. Fabbrichesi, Phys. Rev. D 87, 036001 (2013); M. Farina, D. Pappadopulo, and A. Strumia, J. High Energy Phys. 08 (2013) 022; A. de Gouvea, D. Hernandez, and T. M. P. Tait, Phys. Rev. D 89, 115005 (2014); H. Davoudiasl and I. M. Lewis, Phys. Rev. D 90, 033003 (2014).

[6] P. Minkowski, Phys. Lett. 67B, 421 (1977); R. N. Mohapatra and G. Senjanovic, Phys. Rev. Lett. 44, 912 (1980); T. Yanagida, AIP Conf. Proc. C7902131, 95 (1979); M. Gell-Mann, P. Ramond, and R. Slansky, AIP Conf. Proc. C790927, 315 (1979).
[7] M. Fukugita and T. Yanagida, Phys. Lett. B 174, 45 (1986).

[8] S. Davidson and A. Ibarra, Phys. Lett. B 535, 25 (2002).

[9] G. Giudice, A. Notari, M. Raidal, A. Riotto, and A. Strumia, Nucl. Phys. B685, 89 (2004).

[10] J. Casas and A. Ibarra, Nucl. Phys. B618, 171 (2001).

[11] M. Gonzalez-Garcia, M. Maltoni, and T. Schwetz, J. High Energy Phys. 11 (2014) 052.

[12] P. Frampton, S. Glashow, and T. Yanagida, Phys. Lett. B 548, 119 (2002); M. Raidal and A. Strumia, Phys. Lett. B 553, 72 (2003); W.-1. Guo, Z.-z. Xing, and S. Zhou, Int. J. Mod. Phys. E E16, 1 (2007).

[13] S. Blanchet and P. Di Bari, J. Cosmol. Astropart. Phys. 03 (2007) 018; F. Josse-Michaux and A. Abada, J. Cosmol. Astropart. Phys. 10 (2007) 009.

[14] P. Di Bari, Nucl. Phys. B727, 318 (2005).

[15] O. Vives, Phys. Rev. D 73, 073006 (2006); G. Engelhard, Y. Grossman, E. Nardi, and Y. Nir, Phys. Rev. Lett. 99, 081802 (2007).

[16] A. Pilaftsis and T.E. Underwood, Nucl. Phys. B692, 303 (2004).

[17] E. K. Akhmedov, V. A. Rubakov, and A. Y. Smirnov, Phys. Rev. Lett. 81, 1359 (1998). 


\section{University Library}

\section{- M M I N E R VA A gateway to Melbourne's research publications}

Minerva Access is the Institutional Repository of The University of Melbourne

Author/s:

Clarke, JD;Foot, R;Volkas, RR

Title:

Electroweak naturalness in the three-flavor type I seesaw model and implications for leptogenesis

Date:

2015-04-10

\section{Citation:}

Clarke, J. D., Foot, R. \& Volkas, R. R. (2015). Electroweak naturalness in the three-flavor type I seesaw model and implications for leptogenesis. PHYSICAL REVIEW D, 91 (7), https:// doi.org/10.1103/PhysRevD.91.073009.

Persistent Link:

http://hdl.handle.net/11343/116038 